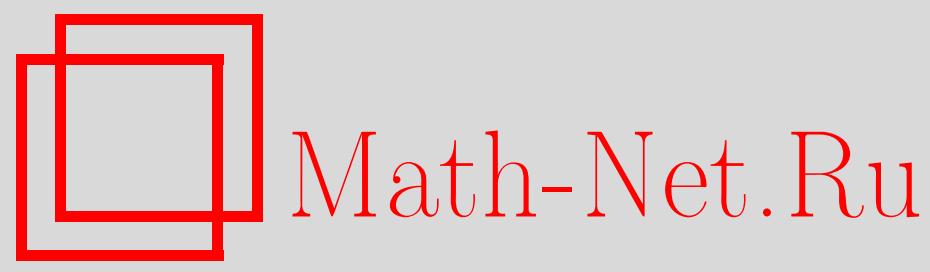

В. А. Ватутин, Е. Е. Дьяконова, Асимптотические свойства многотипных критических ветвящихся процессов, эволюционирующих в случайной среде, Дискрет. матем., 2010, том 22, выпуск 2, 22-40

DOI: https://doi.org/10.4213/dm1092

Использование Общероссийского математического портала Math-Net.Ru подразумевает, что вы прочитали и согласны с пользовательским соглашением http://www . mathnet.ru/rus/agreement

Параметры загрузки:

IP: 18.209.158.208

26 апреля 2023 г., $17: 27: 47$ 


\title{
Асимптотические свойства многотипных критических ветвящихся процессов, эволющионирующих в случайной среде
}

\author{
() 2010 г. В. А. Ватутин, Е. Е. Дьяконова
}

\begin{abstract}
Для расширенного класса многотипных критических ветвящихся процессов, эволюционирующих в случайной среде, найдена асимптотика вероятности невырождения при условиях, которые являются более слабыми, чем даже известные к настоящему времени условия для случая процессов с одним типом частиц. Доказана функциональная предельная теорема для числа частиц в процессе в моменты времени $n t, 0 \leqslant t \leqslant 1$, при условии невырождения процесса к моменту $n$.

Работа выполнена при финансовой поддержке РФФИ (грант 08-01-00078) и программы «Математическая теория управления».
\end{abstract}

\section{1. Введение и основные результаты}

Смит и Вилкинсон [1] первыми предложили исследовать ветвящиеся процессы, эволюционирующие в случайной среде, или коротко, ветвящиеся процессы в случайной среде (ВПСС). В их работе среда порождалась последовательностью независимых одинаково распределенных случайных величин. Затем Атрейя и Карлин [2] проанализировали свойства ветвящихся процессов, находящихся под влиянием случайной среды более общего вида. С тех пор появилось большое количество работ, посвященных исследованию поведения ветвящихся процессов в случайной среде (см., например, [3-18]). Было установлено, что свойства надкритических ветвящихся процессов в случайной среде близки к свойствам классических надкритических ветвящихся процессов Гальтона-Ватсона (см. [19]), в то время, как критические и докритические ветвящиеся процессы в случайной среде обладают рядом особенностей, не имеющих аналогов в поведении классических критических и докритических процессов Гальтона-Ватсона (см. $[6,8,12,14,18])$.

До появления статей [7] и [17] стандартным условием, накладываемым на ВПСС, было предположение о конечности второго момента $\mathbf{E} X^{2}<\infty$ приращения $X$ сопровождающего случайного блуждания. Именно при этом условии (и соответственно, при его многомерном аналоге в многотипном случае) была найдена асимптотика вероятности невырождения критических однотипных ВПСС в [18] и [8], и критических многотипных ВПСС в [15] для среды, порожденной последовательностью независимых одинаково распределенных случайных величин. Понятие критичности ВПСС было расширено в работах [7] и [17], что позволило ввести новую классификацию ВПСС и найти (в рамках так называемого annealed approach) асимптотику вероятности невырождения для гораздо более богатого класса критических однотипных ВПСС. Для процессов, входящих в 
расширенный класс критических однотипных ВПСС, конечность второго момента случайной величины $X$ не обязательна. Более того, даже существование $\mathbf{E} X$ не является необходимым требованием. Статьи [9-11], посвященные изучению однотипного случая (в рамках так называемого quenched approach), также продемонстрировали преимущества новой классификации ВПСС.

Настоящая работа посвящена изучению (в рамках annealed approach) таких многотипных критических ветвящихся процессов в случайной среде, сопровождающее случайное блуждание которых удовлетворяет условию Спитцера-Дони (см. ниже условие А3). В частности, мы обобщаем некоторые результаты статей [7] и [16].

Для описания исследуемой модели ВПСС нам потребуется ряд стандартных векторных обозначений.

Пусть $\mathbf{e}_{j}, j=1, \ldots, p,-p$-мерная вектор-строка, $j$-я компонента которой равняется 1 , а все остальные компоненты нулю, $\mathbf{0}=(0, \ldots, 0)-p$-мерная нулевая вектор-строка.

Для $\mathbf{x}=\left(x_{1}, \ldots, x_{p}\right)$ и $\mathbf{y}=\left(y_{1}, \ldots, y_{p}\right)^{\prime}$ положим

$$
\|\mathbf{x}\|=\sum_{i=1}^{p}\left|x_{i}\right|, \quad(\mathbf{x}, \mathbf{y})=\sum_{i=1}^{p} x_{i} y_{i} .
$$

Пусть $\mathbf{N}_{0}=\{0,1,2, \ldots\}$ и $\mathbf{N}_{0}^{p}-$ множество всех векторов $\mathbf{z}=\left(z_{1}, \ldots, z_{p}\right)$ с неотрицательными целочисленными координатами. Далее, будем обозначать через $\mathbf{J}^{p}$ множество всех вектор-столбцов вида

$$
\mathbf{s}=\left(s_{1}, \ldots, s_{p}\right)^{\prime}, \quad 0 \leqslant s_{i} \leqslant 1, \quad i=1, \ldots, p .
$$

Для $\mathbf{s}=\left(s_{1}, \ldots, s_{p}\right)^{\prime} \in \mathbf{J}^{p}$ и $\mathbf{z}=\left(z_{1}, \ldots, z_{p}\right) \in \mathbf{N}_{0}^{p}$ положим

$$
\mathbf{s}^{\mathbf{z}}=\prod_{i=1}^{p} s_{i}^{z_{i}}
$$

Пусть $(\mathbb{F}, \mathscr{B}(\mathbb{F}))$ - пространство вероятностных мер $\Phi$ на $\mathbf{N}_{0}^{p}$ с $\sigma$-алгеброй $\mathscr{B}(\mathbb{F})$ борелевских множеств, снабженное метрикой расстояния по вариации. Обозначим $\left(\mathbb{F}_{p}, \mathscr{B}_{(}\left(\mathbb{F}_{p}\right)\right)$ прямое $p$-кратное произведение пространства $(\mathbb{F}, \mathscr{B}(\mathbb{F}))$ на себя.

Пусть $\mathbf{F}=\left(F^{(1)}, \ldots, F^{(p)}\right)^{\prime}-$ случайная величина (векторнозначная случайная мера, то есть вектор, компонентами которого являются вероятностные меры), принимающая значения в $\left(\mathbb{F}_{p}, \mathscr{B}\left(\mathbb{F}_{p}\right)\right)$, то есть $\mathbf{F}-$ измеримое отображение некоторого вероятностного пространства $(\Omega, \mathscr{A}, \mathbf{P})$ в $\left(\mathbb{F}_{p}, \mathscr{B}\left(\mathbb{F}_{p}\right)\right)$. Назовем случайной средой $\Pi=\left(\mathbf{F}_{0}, \mathbf{F}_{1}, \mathbf{F}_{2}, \ldots\right)$ бесконечную последовательность независимых одинаково распределенных вероятностных копий случайной величины $\mathbf{F}$, и будем говорить, что $\mathbf{F}$ порождает П.

Ввиду взаимно однозначного соответствия между вероятностными мерами и производящими функциями, будем отождествлять $\mathbf{F}=\left(F^{(1)}, \ldots, F^{(p)}\right)^{\prime}$, порождающую П, со случайным $p$-мерным вектором-столбцом $\mathbf{f}(\mathbf{s})=\left(f^{(1)}(\mathbf{s}), \ldots, f^{(p)}(\mathbf{s})\right)^{\prime}$, компонентами которого служат $p$-мерные (случайные) производящие функции $f^{(i)}$, соответствующие $F^{(i)}, 1 \leqslant i \leqslant p$ :

$$
f^{(i)}(\mathbf{s})=\sum_{\mathbf{z} \in \mathbf{N}_{0}^{p}} F^{(i)}(\{\mathbf{z}\}) \mathbf{s}^{\mathbf{z}}, \quad \mathbf{s} \in \mathbf{J}^{p} .
$$

Аналогично будем отождествлять компоненту $\mathbf{F}_{n}=\left(F_{n}^{(1)}, \ldots, F_{n}^{(p)}\right)^{\prime}, n \geqslant 0$, случайной среды $\Pi=\left(\mathbf{F}_{0}, \mathbf{F}_{1}, \mathbf{F}_{2}, \ldots\right)$ со случайной векторнозначной вероятностной производя- 
щей функцией $\mathbf{f}_{n}(\mathbf{s})=\left(f_{n}^{(1)}(\mathbf{s}), \ldots, f_{n}^{(p)}(\mathbf{s})\right)^{\prime}$, компонентами которой служат (случайные) многомерные вероятностные производящие функции $f_{n}^{(i)}(\mathbf{s})$, соответствующие $F_{n}^{(i)}$, $1 \leqslant i \leqslant p$ :

$$
f_{n}^{(i)}(\mathbf{s})=\sum_{\mathbf{z} \in \mathbf{N}_{0}^{p}} F_{n}^{(i)}(\{\mathbf{z}\}) \mathbf{s}^{\mathbf{z}}, \quad \mathbf{s} \in \mathbf{J}^{p}
$$

Ввиду этого соглашения мы можем формально переписать (1) и (2) в виде

$$
\begin{aligned}
f^{(i)}(\mathbf{s}) & =\mathbf{E}\left[\mathbf{s}^{\xi_{i}} \mid \mathbf{f}\right]=\mathbf{E}\left[s_{1}^{\xi_{i 1}} \ldots s_{p}^{\xi_{i p}} \mid \mathbf{f}\right], & & \mathbf{s} \in \mathbf{J}^{p}, \\
f_{n}^{(i)}(\mathbf{s}) & =\mathbf{E}\left[\mathbf{s}^{\xi_{i}(n)} \mid \mathbf{f}_{n}\right]=\mathbf{E}\left[s_{1}^{\xi_{i 1}(n)} \ldots s_{p}^{\xi_{i p}(n)} \mid \mathbf{f}_{n}\right], & & \mathbf{s} \in \mathbf{J}^{p},
\end{aligned}
$$

где векторы $\xi_{i}=\left(\xi_{i 1}, \ldots, \xi_{i p}\right), i=1, \ldots, p$, при $\left(F^{(1)}, \ldots, F^{(p)}\right)=\left(\Phi^{(1)}, \ldots, \Phi^{(p)}\right)$ распределены согласно мерам $\Phi^{(i)}, i=1, \ldots, p$, и векторы $\xi_{i}(n)=\left(\xi_{i 1}(n), \ldots, \xi_{i p}(n)\right)$, $i=1, \ldots, p$, при условии $\left(F_{n}^{(1)}, \ldots, F_{n}^{(p)}\right)=\left(\Phi_{n}^{(1)}, \ldots, \Phi_{n}^{(p)}\right)$ распределены согласно мерам $\Phi_{n}^{(i)}, i=1, \ldots, p$.

Принимая во внимание описанное выше соответствие, мы часто в дальнейшем будем использовать запись $\Pi=\left(\mathbf{f}_{0}, \mathbf{f}_{1}, \mathbf{f}_{2}, \ldots\right)$ для обозначения среды $\Pi=\left(\mathbf{F}_{0}, \mathbf{F}_{1}, \mathbf{F}_{2}, \ldots\right)$.

Пусть $\left(\Phi_{0}, \Phi_{1}, \ldots\right)$, где

$$
\boldsymbol{\Phi}_{n}=\left(\Phi_{n}^{(1)}, \Phi_{n}^{(2)}, \ldots, \Phi_{n}^{(p)}\right) \in \mathbb{F}_{p}, \quad n=0,1,2, \ldots,
$$

- реализация случайной среды.

Последовательность случайных $p$-мерных векторов $\mathbf{Z}(n)=\left(Z^{(1)}(n), \ldots, Z^{(p)}(n)\right)$, $n=0,1, \ldots$, с неотрицательными целочисленными координатами называется ветвящимся процессом Гальтона-Ватсона с $p$ типами частиц в случайной среде П, если $\mathbf{Z}(0)$ не зависит от П и для всех $n \geqslant 0, \mathbf{z}=\left(z_{1}, \ldots, z_{p}\right) \in \mathbf{N}_{0}^{p}$ и $\boldsymbol{\Phi}_{0}, \boldsymbol{\Phi}_{1}, \ldots \in \mathbb{F}_{p}$ :

$$
\mathscr{L}\left(\mathbf{Z}(n+1) \mid \mathbf{Z}(n)=\left(z_{1}, \ldots, z_{p}\right), \Pi=\left(\boldsymbol{\Phi}_{0}, \boldsymbol{\Phi}_{1}, \ldots\right)\right)=\mathscr{L}\left(\sum_{i=1}^{p} \sum_{j=1}^{z_{i}} \xi_{i}^{(j)}(n)\right),
$$

где случайные $p$-мерные векторы $\xi_{i}^{(1)}(n), \xi_{i}^{(2)}(n), \ldots, \xi_{i}^{\left(z_{i}\right)}(n), i=1, \ldots, p$, имеющие целочисленные неотрицательные координаты, независимы в совокупности, и, кроме того, для каждого $i=1, \ldots, p$ случайные векторы $\xi_{i}^{(1)}(n), \xi_{i}^{(2)}(n), \ldots, \xi_{i}^{\left(z_{i}\right)}(n)$ распределены согласно вероятностной мере $\Phi_{n}^{(i)}$.

Соотношение (3) задает ветвящийся процесс Гальтона-Ватсона в случайной среде, в котором величина $Z^{(i)}(n), i=1, \ldots, p$, представляет собой число частиц типа $i$ в $n$-м поколении, причем частицы в этом процессе эволюционируют следующим образом. Если состояние среды в момент времени $n$ есть $\left(\Phi_{n}^{(1)}, \ldots, \Phi_{n}^{(p)}\right) \in \mathbb{F}_{p}$, то каждая из $Z^{(i)}(n)$ частиц типа $i, i=1, \ldots, p$, существующих в этот момент времени в популяции, производит потомство согласно вероятностной мере $\Phi_{n}^{(i)}$ независимо от размножения других частиц. Таким образом, компонента $Z^{(i)}(n+1)$ вектора $\mathbf{Z}(n+1)=\left(Z^{(1)}(n+1), \ldots, Z^{(p)}(n+1)\right)$ равна числу частиц типа $i$ среди всех непосредственных потомков частиц $n$-го поколения. Начальное распределение вектора $\mathbf{Z}(0)$ будет определено в дальнейшем.

Для $\mathbf{z} \in \mathbf{N}_{0}^{p}, \mathbf{z} \neq \mathbf{0}$, символы $\mathbf{E}_{\mathbf{z}}$ и $\mathbf{P}_{\mathbf{z}}$ будут использоваться для обозначения математического ожидания и, соответственно, вероятности, порождаемых рассматриваемым ветвящимся процессом в случайной среде, в котором $\mathbf{Z}(0)=\mathbf{z}$. Например, $\mathbf{P}_{\mathbf{e}_{j}}$ означает, 
что процесс начался с одной частицы типа $j$. В последующем, чтобы избежать громоздких обозначений, мы будем опускать нижний индекс $\mathbf{z}$ вплоть до формулировки теоремы 3.

Символы $\mathbb{E}_{f}$ и $\mathbb{P}_{f}$ используются для обозначения играющих существенную роль в последующих рассуждениях условного математического ожидания и условной вероятности при фиксированной векторнозначной производящей вероятностной функции $\mathbf{f}=\mathbf{f}(\mathbf{s})=\left(f^{(1)}(\mathbf{s}), \ldots, f^{(p)}(\mathbf{s})\right)^{\prime}, \mathbf{s} \in \mathbf{J}^{p}$, компонентами которой служат многомерные вероятностные производящие функции. Мы также будем использовать символы $\mathbb{E}_{\mathbf{f}}$ и $\mathbb{P}_{\mathbf{f}}$ для обозначения условного математического ожидания и условной вероятности при фиксированной среде $\left(\mathbf{f}_{0}, \mathbf{f}_{1}, \mathbf{f}_{2}, \ldots\right)$.

Положим

$$
m_{i j}=\mathbb{E}_{f} \xi_{i j}, \quad m_{i j}(n)=\mathbb{E}_{\mathbf{f}} \xi_{i j}(n), \quad i, j=1, \ldots, p,
$$

и введем соответствующие матрицы средних

$$
\begin{aligned}
M & =\left(m_{i j}\right)_{i, j=1}^{p}=\left(\mathbb{E}_{f} \xi_{i j}\right)_{i, j=1}^{p}, \\
M_{n} & =\left(m_{i j}(n)\right)_{i, j=1}^{p}=\left(\mathbb{E}_{\mathbf{f}} \xi_{i j}(n)\right)_{i, j=1}^{p}, \quad n \geqslant 0 .
\end{aligned}
$$

Для строго положительных матриц средних $M$ и $M_{n}, n \geqslant 0$, обозначим $\rho$ и $\rho_{n}$ их перроновы корни, то есть их максимальные по абсолютному значению собственные числа.

Перечислим теперь условия, накладываемые на многотипный ветвящийся процесс в случайной среде, которые в дальнейшем мы всегда будем предполагать выполненными.

Условие А1. Матрицы средних $M$ и $M_{n}, n \geqslant 0$, положительны и с вероятностью 1 имеют общий неслучайный положительный правый собственный вектор

$$
\mathbf{u}=\left(u_{1}, \ldots, u_{p}\right)^{\prime}, \quad\|\mathbf{u}\|=1,
$$

такой, что

$$
M \mathbf{u}=\rho \mathbf{u}, \quad M_{n} \mathbf{u}=\rho_{n} \mathbf{u} .
$$

Условие А2. Выполняется соотношение

$$
\mathbf{P}\left(\min _{1 \leqslant i \leqslant p} \mathbb{P}_{f}\left(\left\|\boldsymbol{\xi}_{i}\right\|>1\right)>0\right)=1 .
$$

Накладывая это ограничение, мы тем самым исключаем из анализа случай мер на пространстве вероятностных мер $\left(\mathbb{F}_{p}, \mathscr{B}_{(}\left(\mathbb{F}_{p}\right)\right)$, для которых векторнозначная вероятностная производящая функция $\mathbf{f}(\mathbf{s})$ может с положительной вероятностью иметь компоненты вида

$$
f^{(i)}(\mathbf{s})=p_{0}+p_{i 1} s_{1}+p_{i 2} s_{2}+\ldots+p_{i p} s_{p} .
$$

Это требование носит технический характер, и, по-видимому, может быть ослаблено. Роль этого условия станет ясной из последующих рассуждений.

Пусть

$$
X=\log \rho, \quad X_{n}=\log \rho_{n-1}, \quad n \geqslant 1 .
$$

Положим

$$
S_{0}=0, \quad S_{n}=X_{1}+\ldots+X_{n}, \quad n \geqslant 1 .
$$


Последовательность $S=\left(S_{0}, S_{1}, \ldots\right)$ называется сопровождающим случайным блужданием, соответствующим ветвящемуся процессу $\{\mathbf{Z}(n), n \geqslant 0\}$ в случайной среде П. Известно, что многие важные выводы о свойствах однотипных ветвящихся процессов в случайной среде могут быть сделаны на основе анализа свойств сопровождающих их случайных блужданий (см., например, [7, 17]).

В настоящей работе мы покажем, что аналогичное явление имеет место и для широкого класса многотипных ветвящихся процессов в случайной среде. С этой целью предположим, что сопровождающее случайное блуждание $S$ удовлетворяет условию СпитцераДони (см. [22]).

Условие А3. Существует число $a \in(0,1)$ такое, что при $n \rightarrow \infty$

$$
\mathbf{P}\left(S_{n}>0\right) \rightarrow a
$$

Распространяя расширенную классификацию, введенную для однотипных ветвящихся процессов в случайной среде в [7], мы назовем ветвящийся процесс $\{\mathbf{Z}(n), n \geqslant 0\}$ с $p$ типами частиц и случайной средой П, удовлетворяющий условию А1, критическим, если соответствующее ему сопровождающее случайное блуждание является осциллирующим, то есть если $\limsup _{n \rightarrow \infty} S_{n}=+\infty$ п.н. и $\liminf _{n \rightarrow \infty} S_{n}=-\infty$ п.н. Известно [25], что случайные блуждания, удовлетворяющие условию А3, осциллируют.

Пусть $0=\gamma_{0}<\gamma_{1}<\ldots$ - строгие убывающие лестничные моменты случайного блуждания $S$. Положим

$$
\begin{array}{ll}
V(x)=\sum_{i=0}^{\infty} \mathbf{P}\left(S_{\gamma_{i}} \geqslant-x\right), & x \geqslant 0 ; \\
V(x)=0, & x<0 .
\end{array}
$$

Так как $S$ является осциллирующим, справедливо (см. [21]) соотношение

$$
\mathbf{E} V(x+X)=V(x), \quad x \geqslant 0 .
$$

Для $\beta>0$ положим

$$
\begin{aligned}
\Delta_{i j}(\beta) & =\mathbb{E}_{f}\left|\xi_{i j}-m_{i j}\right|^{\beta}, & \Delta_{i j}(k ; \beta) & =\mathbb{E}_{\mathbf{f}}\left|\xi_{i j}(k-1)-m_{i j}(k-1)\right|^{\beta}, \\
\Delta_{\beta} & =\max _{i, j} \Delta_{i j}(\beta), & \Delta_{\beta}(k) & =\max _{i, j} \Delta_{i j}(k ; \beta)
\end{aligned}
$$

и введем случайные величины

$$
\zeta_{\beta}=e^{-\beta X} \Delta_{\beta}, \quad \zeta_{\beta}(k)=e^{-\beta X_{k}} \Delta_{\beta}(k), \quad k \geqslant 1 .
$$

Условие А4. Существуют числа $\beta \in(1,2]$ и $\varepsilon>0$ такие, что с вероятностью 1

$$
\Delta_{\beta}(k)<\infty, \quad k \geqslant 1,
$$

И

$$
\mathbf{E}\left[\log ^{+} \zeta_{\beta}\right]^{1 / a+\varepsilon}<\infty, \quad \mathbf{E}\left[V(X)\left(\log ^{+} \zeta_{\beta}\right)^{1+\varepsilon}\right]<\infty
$$

где величина $a$ та же, что и в условии А3. 
Введем случайные величины

$$
Q^{(i)}(n)=\mathbb{P}_{\mathbf{f}}\left(\mathbf{Z}(n) \neq \mathbf{0} \mid \mathbf{Z}(0)=\mathbf{e}_{i}\right), \quad \mathbf{Q}(n)=\left(Q^{(1)}(n), \ldots, Q^{(p)}(n)\right),
$$

и пусть

$$
q_{i}(n)=\mathbf{P}\left(\mathbf{Z}(n) \neq \mathbf{0} \mid \mathbf{Z}(0)=\mathbf{e}_{i}\right)
$$

- вероятность невырождения ветвящегося процесса Гальтона-Ватсона в случайной среде с $p$ типами частиц, начавшегося в момент времени $n=0$ с одной частицы типа $i$.

Заметим, что если условия А1 и А3 выполнены, то

$$
\begin{aligned}
(\mathbf{Q}(n), \mathbf{u}) & \leqslant \min _{0 \leqslant k \leqslant n-1}\left\|M_{0} \cdots M_{k} \mathbf{u}\right\| \\
& =\|\mathbf{u}\| \min _{0 \leqslant k \leqslant n-1} \prod_{j=0}^{k} \rho_{j} \leqslant \exp \left\{\min _{0 \leqslant k \leqslant n} S_{k}\right\} \rightarrow 0, \quad \text { P-П.н. }
\end{aligned}
$$

при $n \rightarrow \infty$ и, следовательно, $Q^{(i)}(n) \rightarrow 0$ P-п.н. при $n \rightarrow \infty$ для всех $1 \leqslant i \leqslant p$.

Теорема 1. Пусть выполнень условия А1- A4. Тогда при $n \rightarrow \infty$,

$$
q_{i}(n) \sim c_{i} n^{-(1-a)} l(n), \quad i=1, \ldots, p,
$$

где $c_{i}>0, i=1, \ldots, p$, a l(n) - функиия, медленно меняющаяся на бесконечности.

Для фиксированного начального значения $\mathbf{Z}(0)=\mathbf{z} \in \mathbf{N}_{0}^{p}, \mathbf{z} \neq \mathbf{0}$, и целых чисел $0 \leqslant r \leqslant n$ определим процесс $W_{r, n, \mathbf{z}}=\left\{W_{r, n, \mathbf{z}}(t), 0 \leqslant t \leqslant 1\right\}$ соотношением

$$
W_{r, n, \mathbf{z}}(t)=\frac{(\mathbf{Z}(r+[(n-r) t]), \mathbf{u})}{\exp \left\{S_{r+[(n-r) t]}\right\}}, \quad 0 \leqslant t \leqslant 1 .
$$

Здесь и далее $[x]$ обозначает целую часть числа $x$.

Теорема 2. Пусть выполнень условия А1-A3, и, кроме того, условие А4 с $\beta=2$. Пусть $r_{1}, r_{2}, \ldots$ - последовательность натуральных чисел такая, что $r_{n} \leqslant n u r_{n} \rightarrow \infty$. Тогда при $n \rightarrow \infty$

$$
\mathscr{L}\left(W_{r, n} \mid \mathbf{Z}(n) \neq \mathbf{0} ; \mathbf{Z}(0)=\mathbf{z}\right) \Longrightarrow \mathscr{L}\left(W_{\mathbf{z}}(t), 0 \leqslant t \leqslant 1\right),
$$

где предельный процесс является случайным процессом с п.н. постоянными траекториями, то есть

$$
\mathbf{P}\left(W_{\mathbf{z}}(t)=W_{\mathbf{z}} \text { при всех } t \in[0,1]\right)=1 \text {. }
$$

Более того,

$$
\mathbf{P}\left(0<W_{\mathbf{z}}<\infty\right)=1 \text {. }
$$

Здесь символ $\Longrightarrow$ обозначает сходимость в топологии Скорохода в пространстве $D[0,1]$ функций, определенных на $[0,1]$, непрерывных справа и имеющих левосторонние пределы.

Результаты настоящей работы обобщают и расширяют соответствующие утверждения, установленные в статьях [7] и [16]. Так, теорема 1 является обобщением теоремы 3 из 
[16]. Отметим, что ввиду условия (10) асимптотическое соотношение (13) выполняется, если, например,

$$
\sum_{i, j} \mathbb{E}_{f}\left(\xi_{i j}-m_{i j}\right)^{2}=\infty
$$

с вероятностью 1. Таким образом, даже в случае $p=1$ условия, налагаемые в теореме 1 , являются более слабыми, чем соответствующие условия из следствия 1.2 в [7]. Упомянутое следствие является на сегодняшний день наиболее сильным результатом, описывающим асимптотическое поведение вероятности невырождения однотипных ветвящихся процессов в случайной среде, и оно доказано в предположении конечности вторых моментов распределений численности потомства частиц.

Теорема 2 является обобщением теоремы 1.3 из [7] в двух направлениях. Во-первых, она доказана для процессов с несколькими типами частиц, и, во-вторых, даже для процессов с одним типом частиц условия теоремы 2 слабее условий, накладываемых на характеристики ветвящегося процесса в [7].

\section{2. Вспомогательные результаты}

В дальнейшем, наряду с мерой $\mathbf{P}$, нам понадобится также связанная с ней мера $\mathbf{P}^{+}$. Для описания интересующей нас меры обозначим $\Sigma_{n}$ множество, состоящее из всех возможных наборов $\left(\mathbf{Z}(0), \ldots, \mathbf{Z}(n), \mathbf{f}_{0}, \ldots, \mathbf{f}_{n-1}\right)$, при этом $\Sigma=\Sigma_{\infty}$. Введем $\sigma$-алгебры $\left\{\mathscr{F}_{n}, n \geqslant 0\right\}$, полагая

$$
\mathscr{F}_{0}=\sigma(\mathbf{Z}(0)), \quad \mathscr{F}_{n}=\sigma\left(\mathbf{Z}(0), \ldots, \mathbf{Z}(n), \mathbf{f}_{0}, \ldots, \mathbf{f}_{n-1}\right), \quad n \geqslant 1 .
$$

Заметим, что $\sigma$-алгебра $\mathscr{F}_{0}$ является тривиальной, если начальное значение $\mathbf{Z}(0)$ не случайно.

Пусть

$$
\mathscr{F}=\bigvee_{n} \mathscr{F}_{n},
$$

где $\bar{V}_{n}$ обозначает дополнение $\bigvee_{n} \mathscr{F}_{n}$ до $\sigma$-алгебры. В последующем тройка $(\Sigma, \mathscr{F}, \mathbf{P})$ будет служить нам в качестве основного вероятностного пространства.

Обозначим $I\{C\}$ индикатор события $C$, и пусть $L_{n}=\min \left(S_{0}, \ldots, S_{n}\right)$.

Следуя лемме 2.4 из [7], несложно показать, что последовательность $V\left(S_{n}\right) I\left\{L_{n} \geqslant 0\right\}$, $n=0,1, \ldots$, является мартингалом относительно фильтрации $\left\{\mathscr{F}_{n}, n \geqslant 0\right\}$. Следовательно,

$$
\mathbf{E}\left[V\left(S_{n+1}\right) I\left\{L_{n+1} \geqslant 0\right\} \mid \mathscr{F}_{n}\right]=V\left(S_{n}\right) I\left\{L_{n} \geqslant 0\right\}, \quad \text { Р-п.н. }
$$

Определим вероятностные меры $\mathbf{P}_{n}^{+}$на $\sigma$-алгебрах $\left\{\mathscr{F}_{n}, n \geqslant 0,\right\}$ соотношением

$$
d \mathbf{P}_{n}^{+}=V\left(S_{n}\right) I\left\{L_{n} \geqslant 0\right\} d \mathbf{P} .
$$

Ввиду (14), сужение $\left.\mathbf{P}_{n+1}^{+}\right|_{\mathscr{F}_{n}}$ меры $\mathbf{P}_{n+1}^{+}$на $\mathscr{F}_{n}$ есть $\mathbf{P}_{n}^{+}$. Следовательно, существует вероятностная мера $\mathbf{P}^{+}$на $\mathscr{F}$ такая, что

$$
\left.\mathbf{P}^{+}\right|_{\mathscr{F}_{n}}=\mathbf{P}_{n}^{+}, \quad n \geqslant 0 .
$$


В частности, для любой $\mathscr{F}_{n}$-измеримой неотрицательной случайной величины $Y_{n}$

$$
\mathbf{E}^{+} Y_{n}=\mathbf{E}\left[Y_{n} V\left(S_{n}\right) ; L_{n} \geqslant 0\right]
$$

где $\mathbf{E}^{+}$- математическое ожидание, порождаемое мерой $\mathbf{P}^{+}$.

Используя обозначения (9), введем случайные величины

$$
\varphi_{\beta}(k)=\zeta_{\beta}(k+1) e^{-(\beta-1) S_{k}}=e^{-\beta X_{k+1}} \Delta_{\beta}(k+1) e^{-(\beta-1) S_{k}} .
$$

Лемма 1. При выполнении условий $\mathrm{A} 1, \mathrm{~A} 3$ и $\mathrm{A} 4$

$$
\sum_{k=0}^{\infty} \varphi_{\beta}(k)<\infty \quad \mathbf{P}^{+} \text {-n.н. }
$$

Доказательство. Известно ([7], с. 661), что если выполнено условие А3, то для любого $\delta>0$ найдется число $c>0$ такое, что

$$
S_{k} \geqslant c k^{a-\delta} \quad \mathbf{P}^{+}-\text {П.н. }
$$

Таким образом,

$$
e^{-(\beta-1) S_{k}}=O\left(e^{-k^{a-\delta}}\right) \quad \mathbf{P}^{+} \text {-П.н. }
$$

для любого $\delta>0$.

Далее, в [7] (см. с. 663) было показано, что если выполнены условия А3 и (11), то при достаточно малом $\delta_{1}>0$

$$
\zeta_{\beta}(k)=O\left(e^{-k^{a-\delta_{1}}}\right), \quad \mathbf{P}^{+}-\text {П.н. }
$$

Из (18) и (19) следует (17). Лемма доказана.

Напомним, что согласно определению

$$
e^{S_{0}}=1, \quad e^{S_{n}}=\prod_{i=0}^{n-1} \rho_{i}, \quad n \geqslant 1 .
$$

Имея это в виду, положим

$$
W_{n}=e^{-S_{n}}(\mathbf{Z}(n), \mathbf{u}), \quad n \geqslant 0 .
$$

Лемма 2. Если условие А1 выполнено, то при фиксированной среде $\mathbf{f}_{0}, \mathbf{f}_{1}, \ldots$ последовательность $\left\{W_{n}, n \geqslant 0\right\}$ является мартингалом относительно фильтрации $\left\{\mathscr{F}_{n}, n \geqslant 0\right\}$.

Доказательство. Заметим, что

$$
\begin{aligned}
\mathbb{E}_{\mathbf{f}}\left[W_{n} \mid \mathscr{F}_{n-1}\right] & =\mathbb{E}_{\mathbf{f}}\left[e^{-S_{n}} \sum_{i=1}^{p} Z_{i}(n) u_{i} \mid \mathscr{F}_{n-1}\right] \\
& =\mathbb{E}_{\mathbf{f}}\left[e^{-S_{n}} \sum_{i=1}^{p} u_{i} \sum_{j=1}^{p} \sum_{r=1}^{Z_{j}(n-1)} \xi_{j i}^{(r)}(n-1) \mid \mathscr{F}_{n-1}\right],
\end{aligned}
$$


где $\xi_{j i}^{(r)}(n-1)-$ число частиц типа $i$ среди непосредственных потомков $r$-й частицы типа $j$, живущей в поколении $n-1$. Таким образом,

$$
\begin{aligned}
\mathbb{E}_{\mathbf{f}}\left[W_{n} \mid \mathscr{F}_{n-1}\right] & =e^{-S_{n}} \sum_{i=1}^{p} u_{i} \sum_{j=1}^{p} Z_{j}(n-1) m_{j i}(n-1) \\
& =e^{-S_{n}} \sum_{j=1}^{p} Z_{j}(n-1) \sum_{i=1}^{p} m_{j i}(n-1) u_{i} \\
& =e^{-S_{n}} \rho_{n-1} \sum_{j=1}^{p} Z_{j}(n-1) u_{j}=W_{n-1} .
\end{aligned}
$$

В частности,

$$
\mathbb{E}_{\mathbf{f}}\left[W_{n}\right]=\mathbb{E}_{\mathbf{f}}\left[W_{0}\right], \quad n=0,1, \ldots
$$

Лемма доказана.

Следующее утверждение является частным случаем теоремы 2 статьи [13].

Лемма 3. Пусть $Y_{1}, Y_{2}, \ldots, Y_{n}-$ мартингал, $Y_{0}=0, u T_{k}=Y_{k}-Y_{k-1}, k=1,2, \ldots, n$. Если $\mathbf{E}\left|T_{k}\right|^{\beta}<\infty, k=1,2, \ldots, n$, при некотором $\beta \in(1,2]$, то

$$
\mathbf{E}\left|Y_{n}\right|^{\beta} \leqslant 4 \sum_{k=1}^{n} \mathbf{E}\left|T_{k}\right|^{\beta}
$$

Теперь у нас есть все необходимое для доказательства следующей важной леммы.

Лемма 4. При выполнении условий $\mathrm{A} 1, \mathrm{~A} 3$ и А4

$$
\mathbb{P}_{\mathbf{f}}\left(W_{n}>0 \text { при всех } n \mid \mathbf{Z}(0)\right)>0, \quad \mathbf{P}^{+}-\text {-н.н., }
$$

и, следовательно,

$$
\mathbf{P}^{+}\left(W_{n}>0 \text { при всех } n \mid \mathbf{Z}(0)\right)>0 \text {. }
$$

Доказательство. Ясно, что

$$
W_{n}=\left(W_{n}-W_{n-1}\right)+\left(W_{n-1}-W_{n-2}\right)+\ldots+\left(W_{1}-W_{0}\right)+W_{0},
$$

где $W_{0}=(\mathbf{Z}(0), \mathbf{u})-$ начальное значение, a

$$
W_{k}-W_{k-1}=e^{-S_{k}} \sum_{i=1}^{p} \sum_{j=1}^{p} \sum_{r=1}^{Z_{j}(t-1)} u_{i}\left(\xi_{j i}^{(r)}(k-1)-m_{j i}(k-1)\right) .
$$

Возьмем $\beta \in(1,2]$, для которого выполнено условие (10), и оценим условное математическое ожидание $\mathbb{E}_{\mathbf{f}}\left|W_{k}-W_{k-1}\right|^{\beta}$ при $k=1,2, \ldots, n$. Так как условные математические ожидания случайных величин $u_{i}\left(\xi_{j i}^{(r)}(k-1)-m_{j i}(k-1)\right), r=1, \ldots, Z_{j}(t-1)$, 
$j, i \in\{1, \ldots, p\}$, относительно $\sigma$-алгебры $\mathscr{F}_{k-1}$ независимы и $\Delta_{j i}(k ; \beta)<\infty$ (см. (8)), то согласно неравенству фон Бара-Эссеена [20]

$$
\begin{aligned}
\mathbb{E}_{\mathbf{f}}\left[\left|W_{k}-W_{k-1}\right|^{\beta} \mid \mathscr{F}_{k-1}\right] & \leqslant 2 e^{-\beta S_{k}} \sum_{i=1}^{p} \sum_{j=1}^{p} Z_{j}(t-1) u_{i}^{\beta} \mathbb{E}_{\mathbf{f}}\left|\xi_{j i}^{(r)}(k-1)-m_{j i}(k-1)\right|^{\beta} \\
& =2 e^{-\beta S_{k}} \sum_{i=1}^{p} \sum_{j=1}^{p} Z_{j}(t-1) u_{i}^{\beta} \Delta_{j i}(k ; \beta) \\
& \leqslant 2 e^{-\beta S_{k}} \sum_{i=1}^{p} \sum_{j=1}^{p} Z_{j}(t-1) u_{i} \Delta_{j i}(t ; \beta) .
\end{aligned}
$$

Следовательно, при $u_{*}=\min _{i} u_{i}$ и $u^{*}=\max _{i} u_{i}$

$$
\begin{aligned}
\mathbb{E}_{\mathbf{f}}\left|W_{k}-W_{k-1}\right|^{\beta} & =\mathbb{E}_{\mathbf{f}}\left[\mathbb{E}_{\mathbf{f}}\left[\left|W_{k}-W_{k-1}\right|^{\beta} \mid \mathscr{F}_{k-1}\right]\right] \\
& \leqslant \mathbb{E}_{\mathbf{f}}\left[2 e^{-\beta S_{k}} \sum_{i=1}^{p} \sum_{j=1}^{p} Z_{j}(k-1) u_{i} \Delta_{j i}(k ; \beta)\right] \\
& \leqslant 2 e^{-\beta S_{k}} \Delta_{\beta}(k) \mathbb{E}_{\mathbf{f}}\left[\sum_{i=1}^{p} \sum_{j=1}^{p} Z_{j}(k-1) \frac{u_{i}}{u_{j}} u_{j}\right] \\
& \leqslant \frac{2 p u^{*}}{u_{*}} e^{-\beta S_{k}} \Delta_{\beta}(k) \mathbb{E}_{\mathbf{f}} \sum_{j=1}^{p} Z_{j}(k-1) u_{j} \\
& =\frac{2 p u^{*}}{u_{*}} e^{-\beta X_{k}} \Delta_{\beta}(k) e^{-(\beta-1) S_{k-1} \mathbb{E}_{\mathbf{f}} W_{k-1}=\frac{2 p u^{*}}{u_{*}} \varphi_{\beta}(k-1) \mathbb{E}_{\mathbf{f}} W_{0} .}
\end{aligned}
$$

Так как при фиксированной среде последовательность $\left\{W_{n}, n \geqslant 0\right\}$ является мартингалом относительно фильтрации $\left\{\mathscr{F}_{n}, n \geqslant 0\right\}$, применяя лемму 3 с $T_{k}=W_{k}-W_{k-1}$, получаем, что

$$
\begin{aligned}
\mathbb{E}_{\mathbf{f}} W_{n}^{\beta} & =\mathbb{E}_{\mathbf{f}}\left(\sum_{k=1}^{n} T_{k}+W_{0}\right)^{\beta} \leqslant \mathbb{E}_{\mathbf{f}}\left(\left|\sum_{k=1}^{n} T_{k}\right|+W_{0}\right)^{\beta} \leqslant 2^{\beta}\left(\mathbb{E}_{\mathbf{f}}\left|\sum_{k=1}^{n} T_{k}\right|^{\beta}+\mathbb{E}_{\mathbf{f}} W_{0}^{\beta}\right) \\
& \leqslant 4 \cdot 2^{\beta} \sum_{k=1}^{n} \mathbb{E}_{\mathbf{f}}\left|T_{k}\right|^{\beta}+2^{\beta} \mathbb{E}_{\mathbf{f}} W_{0}^{\beta} \leqslant 2^{\beta+3} \frac{p u^{*} \mathbb{E}_{\mathbf{f}} W_{0}}{u_{*}} \sum_{k=0}^{n-1} \varphi_{\beta}(k)+2^{\beta} \mathbb{E}_{\mathbf{f}} W_{0}^{\beta} .
\end{aligned}
$$

Неравенство Гёльдера для неотрицательной случайной величины $\eta$ и чисел $\beta>1$ и $\gamma^{-1}=\left(1-\beta^{-1}\right)^{-1}$ приводит к оценке

$$
\mathbf{E} \eta=\mathbf{E} \eta I\{\eta>0\} \leqslant\left(\mathbf{E} \eta^{\beta}\right)^{1 / \beta}\left(\mathbf{E} I^{\gamma}\{\eta>0\}\right)^{1 / \gamma}=\left(\mathbf{E} \eta^{\beta}\right)^{1 / \beta} \mathbf{P}^{1 / \gamma}(\eta>0) .
$$

Отсюда следует, что

$$
\mathbf{P}(\eta>0) \geqslant \frac{(\mathbf{E} \eta)^{\gamma}}{\left(\mathbf{E} \eta^{\beta}\right)^{\gamma / \beta}} .
$$


Следовательно,

$$
\mathbb{P}_{\mathbf{f}}\left(W_{n}>0 \mid \mathbf{Z}(0)\right) \geqslant \frac{\left(\mathbb{E}_{\mathbf{f}} W_{n}\right)^{\gamma}}{\left(\mathbb{E}_{\mathbf{f}} W_{n}^{\beta}\right)^{\gamma / \beta}} \geqslant \frac{\left(\mathbb{E}_{\mathbf{f}} W_{0}\right)^{\gamma}}{\left(2^{\beta+3} \frac{p u^{*}}{u_{*}} \mathbb{E}_{\mathbf{f}} W_{0} \sum_{k=0}^{n-1} \varphi_{\beta}(k)+2^{\beta} \mathbb{E}_{\mathbf{f}} W_{0}^{\beta}\right)^{\gamma / \beta}} .
$$

Таким образом,

$$
\mathbb{P}_{\mathbf{f}}\left(W_{n}>0 \mid \mathbf{Z}(0)\right) \geqslant \frac{1}{\left(a_{0} \sum_{k=0}^{n-1} \varphi_{\beta}(k)+b_{0}\right)^{\gamma / \beta}}, \quad \mathbf{P}^{+} \text {-п.н., }
$$

где

$$
a_{0}=\frac{2^{\beta+3} p u^{*}}{u_{*}\left(\mathbb{E}_{\mathbf{f}} W_{0}\right)^{\beta-1}}, \quad b_{0}=\frac{2^{\beta} \mathbb{E}_{\mathbf{f}} W_{0}^{\beta}}{\left(\mathbb{E}_{\mathbf{f}} W_{0}\right)^{\beta}}
$$

Учитывая монотонность знаменателя дроби, стоящей в правой части (24), заключаем, что

$$
\mathbb{P}_{\mathbf{f}}\left(W_{n}>0 \mid \mathbf{Z}(0)\right) \geqslant \frac{1}{\left(a_{0} \sum_{k=0}^{\infty} \varphi_{\beta}(k)+b_{0}\right)^{\gamma / \beta}}, \quad \mathbf{P}^{+} \text {-п.н. }
$$

Отсюда, применяя лемму 1 , получаем

$$
\mathbb{P}_{\mathbf{f}}\left(W_{n}>0 \text { при всех } n \mid \mathbf{Z}(0)\right)>0, \quad \mathbf{P}^{+} \text {-п.н. }
$$

Усредняя это соотношение по мере $\mathbf{P}^{+}$, мы видим, что

$$
\mathbf{P}^{+}\left(W_{n}>0 \text { при всех } n \mid \mathbf{Z}(0)\right)>0 .
$$

Лемма доказана.

Нам также понадобится еще одно понятие, введенное Танни (см. [3], с. 484).

Определение 1. Многотипный ветвящийся процесс в случайной среде, порожденной последовательностью независимых одинаково распределенных случайных величин, называется строго регулярным относительно меры $\mathbf{P}$, если существует натуральное число $k$ такое, что

$$
\mathbf{P}\left(\min _{1 \leqslant i \leqslant p} \mathbb{P}_{\mathbf{f}}\left(\|\mathbf{Z}(k)\|>1 \mid \mathbf{Z}(0)=\mathbf{e}_{i}\right)>0\right)>0 .
$$

Пусть

$$
\begin{aligned}
& v(0)=0, \\
& v(l)=\min \left\{m>v(l-1): S_{m+i} \geqslant S_{m} \text { для всех } i \geqslant 0\right\}, \quad l \geqslant 1,
\end{aligned}
$$

- моменты, являющиеся точками минимумов для следующих за ними частей траектории сопровождающего случайного блуждания $\left\{S_{n}, n \geqslant 0\right\}$, и пусть

$$
\mathbf{Z}^{*}(l)=\mathbf{Z}(v(l)), \quad l=0,1, \ldots .
$$

Следующее наблюдение весьма важно для дальнейших рассуждений. 
Лемма 5. Относительно меры $\mathbf{P}^{+}$процесс $\mathbf{Z}^{*}(l), l=0,1, \ldots$, является многотипным ветвящимся процессом в случайной среде, имеющей независимые одинаково распределенные компоненты.

Доказательство этого утверждения сводится к практически дословному повторению доказательства леммы 2.6 работы [7].

Лемма 6. Если процесс $\mathbf{Z}(n), n=0,1, \ldots$, удовлетворяет условиям А1, А2 и А3, то проиесс $\mathbf{Z}^{*}(l), l=0,1, \ldots$, является строго регулярным относительно меры $\mathbf{P}^{+}$.

Доказательство. Нам нужно указать натуральное число $l$ такое, что

$$
\mathbf{P}^{+}\left(\min _{1 \leqslant i \leqslant p} \mathbb{P}_{\mathbf{f}}\left(\left\|\mathbf{Z}^{*}(l)\right\|>1 \mid \mathbf{Z}^{*}(0)=\mathbf{e}_{i}\right)>0\right)>0 .
$$

Покажем, что в наших условиях это неравенство выполняется уже при $l=1$. Действительно,

$$
\begin{aligned}
\mathbf{P}^{+}\left(\min _{1 \leqslant i \leqslant p} \mathbb{P}_{\mathbf{f}}\left(\left\|\mathbf{Z}^{*}(1)\right\|>1 \mid \mathbf{Z}^{*}(0)=\mathbf{e}_{i}\right)>0\right) \\
\quad=\sum_{j=1}^{\infty} \mathbf{P}^{+}\left(\min _{1 \leqslant i \leqslant p} \mathbb{P}_{\mathbf{f}}\left(\left\|\mathbf{Z}^{*}(1)\right\|>1 \mid \mathbf{Z}^{*}(0)=\mathbf{e}_{i}\right)>0 ; v(1)=j\right) \\
\quad=\sum_{j=l}^{\infty} \mathbf{P}^{+}\left(\min _{1 \leqslant i \leqslant p} \mathbb{P}_{\mathbf{f}}\left(\|\mathbf{Z}(j)\|>1 \mid \mathbf{Z}(0)=\mathbf{e}_{i}\right)>0 ; v(1)=j\right) .
\end{aligned}
$$

Полагая

$$
\begin{aligned}
& A_{j}=\left\{S_{j}-S_{k}<0, k=1,2, \ldots, j-1\right\}, \\
& B_{j}=\left\{S_{j+i} \geqslant S_{j}, i=1,2, \ldots\right\}
\end{aligned}
$$

и замечая (см. доказательство леммы 2.6 в [7]), что при выполнении условия А3

$$
\mathbf{P}^{+}\left(S_{j+i} \geqslant S_{j}, i=1,2, \ldots \mid S_{j}=x\right)=\frac{1}{V(x)},
$$

и что наборы $\left(v(1), \mathbf{f}_{0}, \ldots, \mathbf{f}_{v(1)}\right)$ и $\left(\mathbf{f}_{v(1)+1}, \mathbf{f}_{v(1)+2}, \ldots\right)$ являются независимыми по отношению к мере $\mathbf{P}^{+}$, мы получаем, опираясь на разложение Танака для случайных блужданий (см. [24]) и используя условия А1 и А2, цепочку равенств:

$$
\begin{aligned}
& \mathbf{P}^{+}\left(\min _{1 \leqslant i \leqslant p} \mathbb{P}_{\mathbf{f}}\left(\|\mathbf{Z}(j)\|>1 \mid \mathbf{Z}(0)=\mathbf{e}_{i}\right)>0 ; v(1)=j\right) \\
& =\mathbf{P}^{+}\left(\min _{1 \leqslant i \leqslant p} \mathbb{P}_{\mathbf{f}}\left(\|\mathbf{Z}(j)\|>1 \mid \mathbf{Z}(0)=\mathbf{e}_{i}\right)>0 ; A_{j} B_{j}\right) \\
& =\mathbf{E}^{+}\left[\mathbf{E}^{+}\left[I\left\{\min _{1 \leqslant i \leqslant p} \mathbb{P}_{\mathbf{f}}\left(\|\mathbf{Z}(j)\|>1 \mid \mathbf{Z}(0)=\mathbf{e}_{i}\right)>0 ; A_{j}\right\} I\left\{B_{j}\right\} \mid S_{j}\right]\right] \\
& =\mathbf{E}^{+}\left[I\left\{\min _{1 \leqslant i \leqslant p} \mathbb{P}_{\mathbf{f}}\left(\|\mathbf{Z}(j)\|>1 \mid \mathbf{Z}(0)=\mathbf{e}_{i}\right)>0 ; A_{j}\right\} \frac{1}{V\left(S_{j}\right)}\right] \\
& =\mathbf{E}\left[I\left\{\min _{1 \leqslant i \leqslant p} \mathbb{P}_{\mathbf{f}}\left(\|\mathbf{Z}(j)\|>1 \mid \mathbf{Z}(0)=\mathbf{e}_{i}\right)>0\right\} I\left\{A_{j}\right\} I\left\{L_{j} \geqslant 0\right\}\right] \\
& =\mathbf{E}\left[I\left\{A_{j}\right\} I\left\{L_{j} \geqslant 0\right\}\right]=\mathbf{E}\left[I\left\{A_{j}\right\} V\left(S_{j}\right) I\left\{L_{j} \geqslant 0\right\} \frac{1}{V\left(S_{j}\right)}\right]=\mathbf{P}^{+}(v(1)=j) .
\end{aligned}
$$


Итак, при выполнении наших условий

$$
\mathbf{P}^{+}\left(\min _{1 \leqslant i \leqslant p} \mathbb{P}_{\mathbf{f}}\left(\left\|\mathbf{Z}^{*}(1)\right\|>1 \mid \mathbf{Z}^{*}(0)=\mathbf{e}_{i}\right)>0\right)=\mathbf{P}^{+}(v(1)<\infty)=1 .
$$

Лемма доказана.

Начиная с формулируемой ниже теоремы 3 и вплоть до конца статьи, мы почти всюду (там, где это имеет существенное значение) будем явным образом обозначать зависимость рассматриваемых случайных величин и мер относительно начального значения $\mathbf{Z}(0)=\mathbf{z} \in \mathbf{N}_{0}^{p}, \mathbf{z} \neq \mathbf{0}$. Так, например, в этом случае мы будем использовать обозначение $W_{n, \mathbf{z}}$ для $W_{n}$, а также $\mathbf{E}_{\mathbf{z}}\left(\mathbf{E}_{\mathbf{z}}^{+}\right)$и $\mathbf{P}_{\mathbf{z}}\left(\mathbf{P}_{\mathbf{z}}^{+}\right)$, соответственно, для математических ожиданий и вероятностей, порождаемых ветвящимся процессом в случайной среде, имеющим начальное значение $\mathbf{Z}(0)=\mathbf{z}$.

Теорема 3. Пусть выполнены условия А1-A3 и, кроме того, условие $\mathrm{A} 4$ с $\beta=2$. Тогда nри $n \rightarrow \infty$

$$
W_{n, \mathbf{z}} \rightarrow W_{\mathbf{z}}^{+}, \quad \mathbf{P}_{\mathbf{z}}^{+}-n . H .
$$

где случайная величина $W_{\mathbf{z}}^{+}$такова, что

$$
\left\{W_{\mathbf{z}}^{+}>0\right\}=\{\|\mathbf{Z}(n)\|>0 \text { for all } n\}, \quad \mathbf{P}_{\mathbf{z}}^{+}-n . H .
$$

Доказательство. Так как $W_{n, \mathbf{z}}$ является неотрицательным мартингалом, имеет место сходимость (26). Заметим, что

$$
\mathbf{P}_{\mathbf{z}}^{+}\left(W_{\mathbf{z}}^{+}=0\right) \geqslant \mathbf{P}_{\mathbf{z}}^{+}(\|\mathbf{Z}(n)\| \rightarrow 0, n \rightarrow \infty),
$$

поскольку

$$
\{\|\mathbf{Z}(n)\| \rightarrow 0, n \rightarrow \infty \mid \mathbf{Z}(0)=\mathbf{z}\} \subset\left\{W_{\mathbf{z}}^{+}=0\right\}
$$

Для доказательства неравенства, противоположного (27), нам потребуются более деликатные рассуждения.

Напомним сначала, что $\mathbf{Z}^{*}(l), l=0,1, \ldots$, является строго регулярным многотипным ветвящимся процессом в случайной среде, имеющей независимые одинаково распределенные компоненты. Кроме того, ввиду (25)

$$
\mathbf{P}_{\mathbf{z}}^{+}\left(\mathbb{P}_{\mathbf{f}}\left(\left\|\mathbf{Z}^{*}(l)\right\| \rightarrow 0, l \rightarrow \infty\right)<1\right)=1 .
$$

Отсюда, согласно теореме 9.3 из [3], следует, что

$$
\mathbb{P}_{\mathbf{f}}\left(\left\|\mathbf{Z}^{*}(l)\right\| \rightarrow 0, l \rightarrow \infty\right)+\mathbb{P}_{\mathbf{f}}\left(\left\|\mathbf{Z}^{*}(l)\right\| \rightarrow \infty, l \rightarrow \infty\right)=1, \quad \mathbf{P}_{\mathbf{z}}^{+} \text {-п.н. }
$$

Далее, для фиксированного вектора $\mathbf{e}_{j}$, фиксированной среды $\mathbf{f}$ и $k \in\{0,1, \ldots, n-1\}$ положим

$$
g_{n, k, j}(\lambda)=\mathbb{E}_{\mathbf{f}}\left[e^{-\lambda e^{S_{k} W_{n}}} \mid \mathbf{Z}(k)=\mathbf{e}_{j}\right], \quad \lambda \geqslant 0 .
$$

Заметим, что

$$
\mathbb{E}_{\mathbf{f}}\left[e^{S_{k}} W_{n} \mid \mathbf{Z}(k)=\mathbf{e}_{j}\right]=-g_{n, k, j}^{\prime}(0)=u_{j}
$$


Так как функция $-\ln \left\{e^{-\lambda}+\left(1-e^{-\lambda}\right) e^{-\mu}\right\}$ вполне монотонна по $\mu \geqslant 0$ при каждом фиксированном $\lambda \geqslant 0$, и композиция вполне монотонных функций вполне монотонна (см. [25], гл. XIII, §4), функция

$$
h(\mu)=h_{n, k, j}(\mu)=g_{n, k, j}\left(-\ln \left\{e^{-\lambda}+\left(1-e^{-\lambda}\right) e^{-\mu}\right\}\right), \quad \mu \geqslant 0,
$$

вполне монотонна. Кроме того, $h(0)=1$. Следовательно, $h(\mu)$ является преобразованием Лапласа некоторого вероятностного распределения. Обозначим $\varkappa_{n}$ случайную величину, для которой

$$
\mathbb{E}_{\mathbf{f}} e^{-\mu \varkappa_{n}}=h(\mu), \quad \mu \geqslant 0 .
$$

Соотношение (23) при $\beta=\gamma=2$ дает неравенство

$$
\mathbb{P}_{\mathbf{f}}\left(\varkappa_{n}>0\right) \geqslant \frac{\left(\mathbb{E}_{\mathbf{f}} \varkappa_{n}\right)^{2}}{\mathbb{E}_{\mathbf{f}} \varkappa_{n}^{2}} .
$$

Из (31), (30) и (32) следует, что

$$
\begin{aligned}
& \mathbb{E}_{\mathbf{f}} \varkappa_{n}=-h^{\prime}(0)=-g_{n, k, j}^{\prime}(0)\left(1-e^{-\lambda}\right)=u_{j}\left(1-e^{-\lambda}\right), \\
& \mathbb{E}_{\mathbf{f}} \varkappa_{n}^{2}=h^{\prime \prime}(0)=\mathbb{E}_{\mathbf{f}}\left[e^{2 S_{k}} W_{n}^{2} \mid \mathbf{Z}(k)=\mathbf{e}_{j}\right]\left(1-e^{-\lambda}\right)^{2}+u_{j}\left(1-e^{-\lambda}\right) e^{-\lambda} .
\end{aligned}
$$

Учитывая равенства

$$
\mathbb{P}_{\mathbf{f}}\left(\varkappa_{n}>0\right)=1-\lim _{\mu \rightarrow \infty} h(\mu)=1-g_{n, k, j}(\lambda)
$$

и используя соотношения (33), (34) и (35), приходим к оценке

$$
\begin{aligned}
\mathbb{P}_{\mathbf{f}}\left(\varkappa_{n}>0\right) & =1-g_{n, k, j}(\lambda) \\
& \geqslant\left(\frac{\mathbb{E}_{\mathbf{f}}\left[e^{2 S_{k}} W_{n}^{2} \mid \mathbf{Z}(k)=\mathbf{e}_{j}\right]}{u_{j}^{2}}+\frac{e^{-\lambda}}{\left(1-e^{-\lambda}\right) u_{j}}\right)^{-1} .
\end{aligned}
$$

Опираясь на рассуждения, проводившиеся при доказательстве (22), получаем для $k \in\{0,1, \ldots, n-1\}$ неравенство

$$
\begin{aligned}
\mathbb{E}_{\mathbf{f}}\left[e^{2 S_{k}} W_{n}^{2}\right] & =\mathbb{E}_{\mathbf{f}}\left[e^{2 S_{k}}\left(\sum_{i=k+1}^{n} T_{i}+W_{k}\right)^{2}\right] \\
& \leqslant \frac{2^{5} p u^{*} \mathbb{E}_{\mathbf{f}}\left[e^{S_{k}} W_{k}\right]}{u_{*}} \sum_{i=k}^{n-1} \zeta_{2}(i+1) e^{-\left(S_{i}-S_{k}\right)}+2^{2} \mathbb{E}_{\mathbf{f}}\left[e^{2 S_{k}} W_{k}^{2}\right] .
\end{aligned}
$$

Объединяя соотношения (22), (23) при $\beta=\gamma=2$, (33), (36) и (37), заключаем, что $\mathbf{P}_{\mathbf{z}}^{+}$-п.н.

$$
\begin{aligned}
\mathbb{E}_{\mathbf{f}}\left[e^{-\lambda e^{S_{k} W_{n}} \mid}\right. & \left.\mathbf{Z}(k)=\mathbf{e}_{j}\right] \\
& \leqslant 1-\left(32 \frac{p u^{*} u_{j}}{u_{*}} \sum_{i=k}^{n-1} \zeta_{2}(i+1) e^{-\left(S_{i}-S_{k}\right)}+4 u_{j}^{2}+\frac{4 e^{-\lambda}}{\left(1-e^{-\lambda}\right) u_{j}}\right)^{-1} .
\end{aligned}
$$


Переходя к пределу в соотношении (38) сначала при $n \rightarrow \infty$, а затем при $\lambda \rightarrow \infty$, получаем, что для любого фиксированного $k$ и любого $\mathbf{e}_{j}, j=1,2, \ldots, p$,

$$
\mathbb{P}_{\mathbf{f}}\left(W_{\mathbf{z}}^{+}=0 \mid \mathbf{Z}(k)=\mathbf{e}_{j}\right) \leqslant 1-\frac{1}{a \sum_{i=k}^{\infty} \zeta_{2}(i+1) e^{-\left(S_{i}-S_{k}\right)}+b}, \quad \mathbf{P}_{\mathbf{z}}^{+} \text {-П.н., }
$$

где

$$
a=32 \frac{p\left(u^{*}\right)^{2}}{u_{*}}, \quad b=4\left(u^{*}\right)^{2}
$$

Следовательно, для $\mathbf{i}=\left(i_{1}, \ldots, i_{p}\right)$

$$
\mathbb{P}_{\mathbf{f}}\left(W_{\mathbf{z}}^{+}=0 \mid \mathbf{Z}(k)=\mathbf{i}\right) \leqslant\left(1-\frac{1}{a \sum_{i=k}^{\infty} \zeta_{2}(i+1) e^{-\left(S_{i}-S_{k}\right)}+b}\right)^{\|\mathbf{i}\|}, \quad \mathbf{P}_{\mathbf{z}}^{+}-\text {- .н. }
$$

В частности, $\mathbf{P}_{\mathbf{z}}^{+}$-п.н.

$$
\mathbb{P}_{\mathbf{f}}\left(W_{\mathbf{z}}^{+}=0 \mid \mathbf{Z}^{*}(l)=\mathbf{i}\right) \leqslant\left(1-\frac{1}{a \sum_{i=v(l)}^{\infty} \zeta_{2}(i+1) e^{-\left(S_{i}-S_{v(l)}\right)}+b}\right)^{\|\mathbf{i}\|} .
$$

Заметим, что согласно лемме 2.6 в [7] распределение величины, стоящей в правой части (39), не зависит от $l$. Следовательно, для всех $t \geqslant 0 \mathbf{P}_{\mathbf{z}}^{+}-$п.н. справедлива оценка

$$
\begin{aligned}
\mathbb{P}_{\mathbf{f}}\left(W_{\mathbf{z}}^{+}=0\right) & =\mathbb{E}_{\mathbf{f}}\left[\mathbb{P}_{\mathbf{f}}\left(W_{\mathbf{z}}^{+}=0 \mid \mathbf{Z}^{*}(l)\right)\right] \\
& \leqslant \mathbb{E}_{\mathbf{f}}\left[\left(1-\frac{1}{a \sum_{i=v(l)}^{\infty} \zeta_{2}(i+1) e^{-\left(S_{i}-S_{v(l)}\right)}+b}\right)^{\left\|\mathbf{Z}^{*}(l)\right\|}\right] \\
& \leqslant \mathbb{P}_{\mathbf{f}}\left(\left\|\mathbf{Z}^{*}(l)\right\| \leqslant t\right)+\left(1-\frac{1}{a \sum_{i=v(l)}^{\infty} \zeta_{2}(i+1) e^{-\left(S_{i}-S_{v(l)}\right)}+b}\right)^{t} .
\end{aligned}
$$

Усредняя (40) по мере $\mathbf{P}_{\mathbf{z}}^{+}$, переходя к пределу при $l \rightarrow \infty$ и используя (29), получаем, что

$$
\mathbf{P}_{\mathbf{z}}^{+}\left(W_{\mathbf{z}}^{+}=0\right) \leqslant \mathbf{P}_{\mathbf{z}}^{+}\left(\left\|\mathbf{Z}^{*}(l)\right\| \rightarrow 0, l \rightarrow \infty\right)+\mathbf{E}_{\mathbf{z}}^{+}\left[1-\frac{1}{a \sum_{i=0}^{\infty} \varphi_{\beta}(i)+b}\right]^{t} .
$$

Переходя к пределу при $t \rightarrow \infty$, получаем соотношение

$$
\mathbf{P}_{\mathbf{z}}^{+}\left(W_{\mathbf{z}}^{+}=0\right)-\mathbf{P}_{\mathbf{z}}^{+}\left(\left\|\mathbf{Z}^{*}(l)\right\| \rightarrow 0, l \rightarrow \infty\right) \leqslant \mathbf{P}_{\mathbf{z}}^{+}\left(\sum_{i=0}^{\infty} \varphi_{\beta}(i)=\infty\right)=0 .
$$

Таким образом,

$$
\mathbf{P}_{\mathbf{z}}^{+}\left(W_{\mathbf{z}}^{+}=0\right)=\mathbf{P}_{\mathbf{z}}^{+}\left(\left\|\mathbf{Z}^{*}(l)\right\| \rightarrow 0, l \rightarrow \infty\right)=\mathbf{P}_{\mathbf{z}}^{+}(\|\mathbf{Z}(n)\| \rightarrow 0, n \rightarrow \infty) .
$$

Теорема 3 доказана. 


\section{3. Доказательства теорем 1 и 2}

Для $0 \leqslant k \leqslant n$ положим

$$
L_{k, n}=\min _{0 \leqslant j \leqslant n-k}\left(S_{k+j}-S_{k}\right), \quad L_{n}=L_{0, n}=\min \left(S_{0}, \ldots, S_{n}\right),
$$

и пусть

$$
\tau_{n}=\min \left\{0 \leqslant i \leqslant n: S_{i}=L_{n}\right\}
$$

- самая левая точка, в которой случайное блуждание $S_{0}, \ldots, S_{n}$ на интервале $[0, n]$ достигает минимума.

Следующие два результата служат основой при доказательстве теорем 1 и 2.

Лемма 7. Предположим, что выполнено условие А3. Пусть $k \in \mathbf{N}_{0}$, a $Y_{k}-$ ограниченная действительная $\mathscr{F}_{k}$-измеримая случайная величина. Тогда для любого $\mathbf{z} \in \mathbf{N}_{0}^{p}, \mathbf{z} \neq \mathbf{0}$, при $n \rightarrow \infty$,

$$
\mathbf{E}_{\mathbf{z}}\left[Y_{k} \mid L_{n} \geqslant 0\right] \rightarrow \mathbf{E}_{\mathbf{z}}^{+} Y_{k}
$$

Более того, если $Y_{n}, n \in \mathbf{N}_{0}$, - последовательность равномерно ограниченных действительных $\left\{\mathscr{F}_{n}, n \geqslant 0\right\}$-согласованных случайных величин, сходящаяся $\mathbf{P}_{\mathbf{z}}^{+}-$п.н. к некоторой случайной величине $Y_{\infty}$, то при $n \rightarrow \infty$

$$
\mathbf{E}_{\mathbf{z}}\left[Y_{n} \mid L_{n} \geqslant 0\right] \rightarrow \mathbf{E}_{\mathbf{z}}^{+} Y_{\infty}
$$

Лемма 8. Предположим, что выполнено условие А3. Пусть $V_{1}, V_{2}, \ldots$ - последовательность равномерно ограниченных действительных случайных величин таких, что для каждого $k \geqslant 0 u \mathbf{z} \in \mathbf{N}_{0}^{p}, \mathbf{z} \neq \mathbf{0}$,

$$
\mathbf{E}_{\mathbf{z}}\left[V_{n} ; \mathbf{Z}(k) \neq \mathbf{0}, L_{k, n} \geqslant 0 \mid \mathscr{F}_{k}\right]=\mathbf{P}\left(L_{n} \geqslant 0\right)\left(V_{k, \infty}+o(1)\right), \quad \mathbf{P}_{\mathbf{z}}-\text { n.H. }
$$

при $n \rightarrow \infty$ для некоторой последовательности случайных величин $V_{1, \infty}=V_{1, \infty}(\mathbf{z})$, $V_{2, \infty}=V_{2, \infty}(\mathbf{z}), \ldots$. Тогда при $n \rightarrow \infty$

$$
\mathbf{E}_{\mathbf{z}}\left[V_{n} ; \mathbf{Z}\left(\tau_{n}\right) \neq \mathbf{0}\right]=\mathbf{P}\left(L_{n} \geqslant 0\right)\left(\sum_{k=0}^{\infty} \mathbf{E}_{\mathbf{z}}\left[V_{k, \infty} ; \tau_{k}=k\right]+o(1)\right),
$$

где ряд в правой части является абсолютно сходящимся.

Для случая $p=1$ леммы 7 и 8 были установлены в статье [7]. Анализ аргументов, приведенных при доказательстве этих лемм в указанной работе, показывает, что те же самые рассуждения справедливы и для любого $p \geqslant 1$.

Теперь, опираясь на теорему 3 и леммы 7-8, можно убедиться в справедливости утверждений теорем 1 и 2, следуя канве доказательств теорем 1.1 и 1.3 работы [7]. Мы приведем здесь соответствующие аргументы только для полноты изложения.

Доказательство теоремы 1. Для $\mathbf{z} \in \mathbf{N}_{0}^{p}, \mathbf{z} \neq \mathbf{0}$, и $n \in \mathbf{N}_{0}$ введем функцию

$$
\psi(\mathbf{z}, n)=\mathbf{P}_{\mathbf{z}}\left(\mathbf{Z}(n) \neq \mathbf{0}, L_{n} \geqslant 0\right) .
$$


Полагая

$$
\begin{aligned}
Y_{n} & =I\{\mathbf{Z}(n) \neq \mathbf{0}\}, \\
Y_{\infty} & =I\{\mathbf{Z}(n) \neq \mathbf{0} \text { при всех } n \geqslant 0\}
\end{aligned}
$$

в лемме 7 , мы видим, что при $n \rightarrow \infty$ для $\mathbf{z} \neq 0$

$$
\psi(\mathbf{z}, n) \sim \mathbf{P}\left(L_{n} \geqslant 0\right) \mathbf{E}_{\mathbf{z}}^{+}\left[Y_{\infty}\right] .
$$

Известно [23], что при $n \rightarrow \infty$ при выполнении условия А3

$$
\mathbf{P}\left(L_{n} \geqslant 0\right) \sim n^{-(1-a)} l(n),
$$

где $l(n)$ - медленно меняющаяся на бесконечности функция. Положим

$$
V_{n}=I\{\mathbf{Z}(n) \neq \mathbf{0}\} .
$$

Ясно, что при $0 \leqslant k \leqslant n$

$$
\mathbf{E}_{\mathbf{z}}\left[V_{n}, L_{k, n} \geqslant 0 \mid \mathscr{F}_{k}\right]=\psi(\mathbf{Z}(k), n-k), \quad \mathbf{P}_{\mathbf{z}}-\text { П.н. },
$$

где величина $L_{k, n}$ определена в (41). Из (45) и (47) следует, что случайные величины $V_{n}$, $n \geqslant 0$, и

$$
V_{k, \infty}=\mathbf{P}_{\mathbf{Z}(k)}^{+}(\mathbf{Z}(n) \neq \mathbf{0}, n=0,1,2, \ldots)=\mathbf{E}_{\mathbf{Z}(k)}^{+}\left[Y_{\infty}\right], \quad k \geqslant 0,
$$

удовлетворяют условиям леммы 8 . Значит, при $n \rightarrow \infty$

$$
q_{i}(n)=\mathbf{P}_{\mathbf{e}_{i}}(\mathbf{Z}(n) \neq \mathbf{0}) \sim \theta_{i} \mathbf{P}\left\{L_{n} \geqslant 0\right\},
$$

где

$$
\theta_{i}=\sum_{k=0}^{\infty} \mathbf{E}_{\mathbf{e}_{i}}\left[\mathbf{E}_{\mathbf{Z}(k)}^{+}\left[Y_{\infty}\right] ; \tau_{k}=k\right]<\infty,
$$

величина $\tau_{k}$ определена в (42). Заметим, что $\theta_{i}>0$, так как в силу леммы 4

$$
\mathbf{P}_{\mathbf{Z}}^{+}(\mathbf{Z}(n) \neq \mathbf{0} \text { при всех } n \geqslant 0)>0
$$

для любого $\mathbf{z} \neq \mathbf{0}$. Теорема 1 доказана.

Доказательство теоремь 2. Пусть $\phi-$ ограниченная непрерывная функция на пространстве $D[0,1]$ функций, определенных на $[0,1]$, непрерывных справа и имеющих левосторонние пределы. Для $s \in \mathbf{R}$ и $\mathbf{z} \in \mathbf{N}_{0}^{p}, \mathbf{z} \neq \mathbf{0}$, положим

$$
W^{s}(t)=W_{\mathbf{z}}^{s}(t)=e^{-s} W_{\mathbf{z}}^{+}, \quad 0 \leqslant t \leqslant 1,
$$

где случайная величина $W_{\mathbf{z}}^{+}$взята из теоремы 3 . Согласно теореме 3 , для $r_{n} \leqslant n$, фиксированных $s \in \mathbf{R}$ и $\mathbf{z} \in \mathbf{N}_{0}^{p}, \mathbf{z} \neq \mathbf{0}$, процесс $e^{-s} W_{r_{n}, n, \mathbf{z}}$ сходится к $W_{\mathbf{z}}^{s}$ в равномерной метрике при $r_{n} \rightarrow \infty$. Следовательно, в метрике Скорохода на пространстве $D[0,1]$ при $n \rightarrow \infty$ имеет место сходимость

$$
\begin{aligned}
Y_{n} & =\phi\left(e^{-s} W_{r_{n}, n, \mathbf{z}}\right) I\{\mathbf{Z}(n) \neq \mathbf{0} ; \mathbf{Z}(0)=\mathbf{z}\} \\
& \rightarrow Y_{\infty}=\phi\left(W_{\mathbf{z}}^{s}\right) I\left\{W_{\mathbf{z}}^{+}>0\right\}, \quad \mathbf{P}_{\mathbf{z}}^{+} \text {-П.Н. }
\end{aligned}
$$


Для $\mathbf{z} \in \mathbf{N}_{0}^{p}, \mathbf{z} \neq \mathbf{0}$, и $r \leqslant n$ введем функцию

$$
\psi(\mathbf{z}, s, r, n)=\mathbf{E}_{\mathbf{z}}^{+}\left[\phi\left(e^{-s} W_{r, n, \mathbf{z}}\right) ; \mathbf{Z}(n) \neq \mathbf{0}, L_{n}>0\right] .
$$

Применяя лемму 7, получаем равенство

$$
\psi\left(\mathbf{z}, s, r_{n}, n\right)=\mathbf{P}\left(L_{n}>0\right)\left(\mathbf{E}_{\mathbf{z}}^{+}\left[\phi\left(W_{\mathbf{z}}^{s}\right) ; W_{\mathbf{z}}^{+}>0\right]+o(1)\right) .
$$

Ясно, что для $k \leqslant r \leqslant n$

$$
\mathbf{E}_{\mathbf{z}}^{+}\left[\phi\left(W_{r, n, \mathbf{z}}\right) ; \mathbf{Z}(n) \neq \mathbf{0}, L_{k, n} \geqslant 0 \mid \mathscr{F}_{k}\right]=\psi\left(\mathbf{Z}(k), S_{k}, r-k, n-k\right), \quad \mathbf{P}_{\mathbf{z}}^{+} \text {-п.н. }
$$

Следовательно, мы можем применить лемму 8 к случайным величинам

$$
\begin{aligned}
V_{n} & =\phi\left(W_{r_{n}, n, \mathbf{z}}\right) I\{\mathbf{Z}(n) \neq \mathbf{0} ; \mathbf{Z}(0)=\mathbf{z}\}, \\
V_{k, \infty} & =\mathbf{E}_{\mathbf{Z}(k)}^{+}\left[\phi\left(W^{S_{k}}\right) ; W^{+}>0\right] .
\end{aligned}
$$

Воспользовавшись теперь теоремой 1 , получаем, что при $n \rightarrow \infty$

$$
\mathbf{E}_{\mathbf{z}}^{+}\left[\phi\left(W_{r_{n}, n, \mathbf{z}}\right) \mid \mathbf{Z}(n) \neq \mathbf{0}\right] \rightarrow \int \phi(\omega) \lambda_{\mathbf{z}}(d \omega) .
$$

Здесь $\lambda_{\mathbf{z}}-$ мера на пространстве функций $D[0,1]$, определяемая соотношением

$$
\lambda_{\mathbf{z}}(d \omega)=\frac{\sum_{k=0}^{\infty} \mathbf{E}_{\mathbf{z}}\left[\lambda_{\mathbf{Z}(k), S_{k}}(d \omega) ; \mathbf{Z}(k) \neq \mathbf{0}, \tau_{k}=k\right]}{\sum_{k=0}^{\infty} \mathbf{E}_{\mathbf{z}}\left[\mathbf{P}_{\mathbf{Z}(k)}^{+}\left[Y_{\infty}\right] ; \tau_{k}=k\right]},
$$

где

$$
\lambda_{\mathbf{z}, s}(d \omega)=\mathbf{P}_{\mathbf{z}}^{+}\left[W_{\mathbf{z}}^{s} \in d \omega, W_{\mathbf{z}}^{+}>0\right],
$$

а величина $Y_{\infty}$ введена в (44). В силу теоремы 3, мера $\lambda_{\mathbf{z}, s}$ имеет массу $\mathbf{P}_{\mathbf{z}}^{+}\left(W_{n}>0, n=0,1, \ldots\right)$. Таким образом, $\lambda_{\mathbf{z}}$ является вероятностной мерой. Из теоремы 3 вытекает, что мера $\lambda_{\mathbf{z}, s}$ целиком сосредоточена на множестве строго положительных постоянных функций. Этот же факт справедлив и для меры $\lambda_{z}$. Теорема 2 доказана.

\section{Список литературы}

1. Smith W. L., Wilkinson W., On branching processes in random environment. Ann. Math. Statist. (1969) 40, №3, 814-827.

2. Athreya K. B., Karlin S., On branching processes with random environments: I, II. Ann. Math. Statist. (1971) 42, №5-6, 1499-1520, 1843-1858.

3. Tanny D., On multitype branching processes in a random environment. Adv. Appl. Probab. (1981) 13, №3, 464-497.

4. Weissener E. W., Multitype branching processes in random environments. J. Appl. Probab. (1971) 8, №1, 17-31.

5. Kaplan N., Some results about multidimensional branching processes with random environments. Ann. Probab. (1974) 2, №3, 441-455.

6. Афанасьев В. И., Предельная теорема для критического ветвящегося процесса в случайной среде. Дискретная математика (1993) 5, №1, 45-58. 
7. Afanasyev V. I., Geiger J., Kersting G., Vatutin V. A., Criticality for branching processes in random environment. Ann. Probab. (2005) 33, №2, 645-673.

8. Geiger J., Kersting G., The survival probability of a critical branching process in random environment. Теория вероятностей и ее применения (2000) 45, №3, 607-615.

9. Ватутин В. А., Дьяконова Е. Е., Ветвящиеся процессы Гальтона-Ватсона в случайной среде, I: предельные теоремы. Теория вероятностей и ее применения (2003) 48, №2, 274-300.

10. Ватутин В. А., Дьяконова Е. Е., Ветвящиеся процессы Гальтона-Ватсона в случайной среде, II: конечномерные распределения. Теория вероятностей и ее применения (2004) 49, №2, 231-268.

11. Ватутин В. А., Дьяконова Е. Е., Ветвящиеся процессы в случайной среде и бутылочные горлышки в эволюции популяций. Теория вероятностей и ее применения (2006) 51, №1, 22-46.

12. Vatutin V. A., Kyprianou A. E., Branching processes in random environment die slowly. In: Proc. DMTCS, AI, 2008, pp. 375-396.

13. Ватутин В. А., Топчий В. А., Максимум критических процессов Гальтона-Ватсона и непрерывные слева случайные блуждания. Теория вероятностей и ее применения (1997) 42, №1, 21-34.

14. Ватутин В. А., Вахтель В. И., Внезапное вырождение критического ветвящегося процесса в случайной среде. Теория вероятностей и ее применения (2009) 54, №3, 417-438.

15. Дьяконова Е. Е., Об асимптотике вероятности невырождения многомерного ветвящегося процесса в случайной среде. Дискретная математика (1999) 11, №1, 113-128.

16. Дьяконова Е. Е., Критические многотипные ветвящиеся процессы в случайной среде. Дискретная математика (2007) 19, №4, 23-41.

17. Dyakonova E. E., Geiger J., Vatutin V. A., On the survival probability and a functional limit theorem for branching processes in random environment. Markov Process. Related Fields (2004) 10, №2, 289-306.

18. Козлов М. В., Об асимптотике вероятности невырождения критических ветвящихся процессов в случайной среде. Теория вероятностей и ее применения (1976) 21, №4, 813-825.

19. Athreya K. B., Ney P. E., Branching Processes. Springer, Berlin, 1972.

20. von Bahr B., Esseen C.-G., Inequalities for the $r$ th moment of a sum of random variables, $1 \leqslant r \leqslant 2$. Ann. Math. Statist. (1965) 3, 299-303.

21. Bertoin J., Doney R. A., On conditioning a random walk to stay nonnegative. Ann. Probab. (1994) 22, 2152-2167.

22. Doney R. A., Spitzer's condition and ladder variables in random walks. Theory Probab. Related Fields (1995) 101, 577-580.

23. Рогозин Б. А., Распределение первого лестничного момента и высоты и флуктуации случайного блуждания. Теория вероятностей и ее применения (1971) 16, №4, 593-613.

24. Tanaka H., Time reversal of random walks in one dimension. Tokyo J. Math. (1989) 12, 159-174.

25. Феллер В., Введение в теорию вероятностей и ее приложения, 2. Мир, Москва, 1984.

Статья поступила 24.08.2009. 